\title{
Pulsed Digital Oscillators
}

\author{
M. Domínguez*, J. Ricart, J. Pons, F. Rubio \\ MNT Micro and Nano Technologies Group. UPC-Campus Nord Ed. C4 \\ Jordi Girona, 1-3, 08034 Barcelona, Spain. \\ *e-mail:mpumar@eel.upc.edu
}

\begin{abstract}
The objective of this paper is to collect the main latest results on Pulsed Digital Oscillators. Rigorous and experimental results are presented that show what kind of sequences are at their output, the relation between these sequences and those of first-order sigma-delta modulators and how is their performance in practical applications. A new variation of the standard topology of PDOs, on which the feedback variable is not position but the velocity of the resonator, is also presented. The first preliminary results obtained with a PDO working with a MEMS cantilever for chemical sensing are presented, which show that it is possible to infer the oscillation frequency directly from the bitstream at the output of the oscillator. Finally, the dependence of the oscillation frequency as a function of the initial conditions of the resonator are also analyzed with simulations.
\end{abstract}

\section{List of symbols}

$$
\langle a\rangle \quad a \text { Modulus } 1, a \in \mathbb{R}
$$$$
\operatorname{sgn}(b) \quad \operatorname{sign}(\operatorname{Real}(b)), b \in \mathbb{C}
$$

\section{INTRODUCTION}

Pulsed Digital Oscillators (PDOs) are simple circuits that can overcome some of the usual nonlinearities in actuation/sensing of MEMS (Microelectromechanical systems) resonators, [1]-[2]. Their general circuit topology can be seen in Figure 1. PDOs are sampled circuits: at each sampling time it is only necessary to know whether the resonator is above or below its rest position or velocity. The actuation force of this impulsive system, consists of a train of short pulses taking values $\{+F,-F\}$ or $\{+F, 0\}$. Pulsed actuation is a common actuation scheme widely used for MEMS, [5]-[8]. Some works have been directed towards finding optimal pulse wafeforms, [5]. The main advantage of this kind of acutation is that it can overcome most of the typical nonlinearity effects of MEMS actuation forces (electrostatic, thermoelectric, etc.).

The output of the system is the bitstream provided by the 1-bit quantizer (the sign of the sampled position of the resonator). The main features of this structure are the following:

- Simple MEMS drive method: short force pulses of constant amplitude are fed into the resonator.

- Simple MEMS detection requirements: it is only necessary to know if the resonator is above or below its rest (or zero) position/velocity.

- Built-in A/D conversion: the oscillation frequency can be extracted from the bit stream output.

- It works either below or above the Nyquist limit. In the last case, aliasing makes impossible to infer

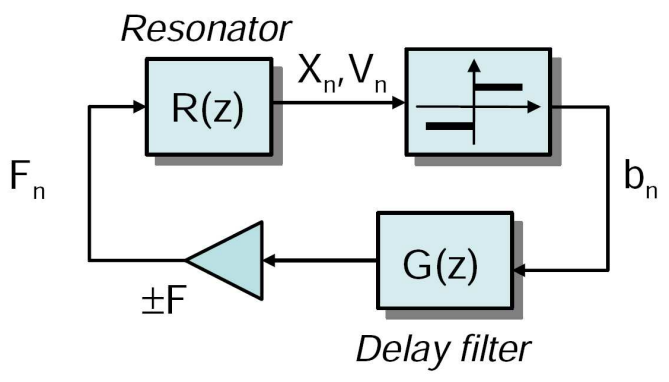

Fig. 1. PDOs general circuit topology.

the exact oscillation frequency directly from the bitstream, but small frequency shifts can be detected.

- For high damping losses of the MEMS resonator, the oscillator response becomes a distorted version of the Devil's staircase fractal. This frequency locking phenomenon is similar to what happens in first-order sigma-delta modulators using leaky integration.

- For a given sampling frequency and feedback sign ( $\delta=1$ or $\delta=-1$ in Figure 1 ), the oscillator either provides energy to the resonator in average, or extracts energy until a limit cycle around the origin is achieved.

The main purpose of this paper is to present rigorous and experimental results that show how these highly nonlinear circuits work, and the latest results obtained results. Let us for a moment look to Figure 1, and assume that the oscillator is working 'well'. It would mean that the resonator position or velocity waveform would be a sinusoid, and therefore the bitstream at the output of the PDO would be the Sign of a Sampled Sinusoid, from here on $S S S=S^{3}$ sequences, [2]:

$$
s(n)=\operatorname{sign}(\sin (2 \pi f n+2 \pi \lambda)), \quad n \geq 0
$$

with $\lambda \in[0,1)$, and being $f \in[0,1 / 2)$ a given normalized frequency. We will consider in this paper that $G(z)=z^{-L}$, and a 1-D mass-spring model for the MEMS resonator:

$$
x^{\prime \prime}(t)+2 \rho \omega_{0} x^{\prime}(t)+\omega_{0}^{2} x(t)=f(t)
$$

where $0<\rho \leq 1$ is the damping factor, $\omega_{0}=2 \pi f_{0}$ is the natural frequency of the resonator and $f(t)$ is the applied external force. 
The evolution of the Pulsed Digital Oscillator can be seen as a discrete-time dynamical system, or more precisely as an iterative map. In this sense, we may define the sequence, $\left\{x_{n}, v_{n}\right\}=\left\{x\left(n T_{s}\right), v\left(n T_{s}\right)\right\}$, of the sampled position and velocity of the resonator, where $T_{s}$ is the sampling period. By an adequate substitution we may construct a second sequence of complex numbers where:

$$
u_{n}=x_{n}+\frac{j}{\sqrt{1-\rho^{2}}}\left(\rho x_{n}+\frac{v_{n}}{\omega_{0}}\right), \quad u_{n} \in \mathbb{C}
$$

and then the evolution of the oscillator is governed by the following simple expression, for $n \geq 0$ :

$$
u_{n+1}=p u_{n}-j \delta F b_{n-L}
$$

being $b_{n}=\operatorname{sgn}\left(u_{n}\right), \delta= \pm 1$, depending on the sign of the feedback loop of the oscillator, and $p=\alpha e^{-j 2 \pi f}$, $\alpha=e^{-\rho \omega_{0}}$, with $f$ the resonant frequency of the resonator. Due to the fact that $F$ is only a scaling factor we will consider, without loss of generality from now on that $F=1$.

In order to consider this system as a map $(\tau)$, we must define our phase space. Let $\mathcal{B}=\{+1,-1\} \subset \mathbb{R}$. The most straightforward option for $L>0$ is:

$$
\begin{array}{rcc} 
& \tau & \\
\mathbb{C} \times \mathcal{B}^{L} & \rightarrow & \mathbb{C} \times \mathcal{B}^{L} \\
\left(u, b_{1}, \cdots, b_{L}\right) & & \left(p u-j \delta b_{L}, \operatorname{sgn}(u), b_{1}, \cdots, b_{L-1}\right)
\end{array}
$$

where $u \in \mathbb{C}, b_{i} \in \mathcal{B}, 1 \leq i \leq L$. For $L=0$ :

$$
\begin{array}{cccc}
\tau: & \mathbb{C} & \rightarrow & \mathbb{C} \\
& u & & p u-j \delta \operatorname{sgn}(u)
\end{array}
$$

Finally we further define:

Definition 1: The projection $\pi_{0}: \mathbb{C} \times \mathcal{B}^{L} \rightarrow \mathbb{C}$ is defined as $\pi_{0}\left(\left(u, b_{1}, \cdots, b_{L}\right)\right)=u$.

Definition 2: The function $T_{n}(z): \mathbb{C} \times \mathcal{B}^{L} \rightarrow \mathbb{C}, n \geq 0$ is defined as $\pi_{0}\left(\tau^{n}(z)\right)$.

It will be shown in Section II that $S^{3}$ sequences are closely related to those at the output of first-order sigmadelta modulators. Sufficient conditions for the oscillation at the resonant frequency of the resonator will be shown in Section III. Finally, in Section IV experimental results are presented in which the PDO is used to monitor changes in the resonant frequency of a chemical sensor.

\section{FIRST ORDER $\Sigma-\Delta$ MODULATORS AND $S^{3}$ BITSTREAMS}

The objective of this section is to establish the relation between the Sign of a Sampled Sinusoid sequences and first-order sigma-delta sequences. $S^{3}$ sequences can be described as (1), and also in the following way:

$$
\frac{s(n)}{2}=\langle n f-1 / 2+\lambda\rangle-\langle n f+\lambda\rangle, \quad n \geq 0
$$

On the other hand, first-order sigma-delta modulators produce sequences of the form [9]:

$$
b(n)=x+\langle(n-1) x+\lambda\rangle-\langle n x+\lambda\rangle, \quad n \geq 0
$$

for some given parameters $x, \lambda \in[0,1)$. The set of all sigma-delta sequences will be named $\mathcal{S D}$. The set of all unordered pairs $\{s,-s\}$ of $S^{3}$ sequences, will be named $\mathcal{S}^{3}$. It may be proved that, [2]:

Theorem 1: A bijection exists between the set $\mathcal{S}^{3}$ and the set of first-order sigma-delta sequences, $\mathcal{S D}$.

For any given sequence $s$, such that $\{s,-s\} \in \mathcal{S}^{3}$, we may generate the sequence $b$ as $b(n)=$ $\frac{1}{2}|s(n)-s(n-1)|$, and $b \in \mathcal{S D}$. Conversely, given a sequence $b \in \mathcal{S D}$, the sequence $s$ generated as $s(0)=1$ (or $s(0)=-1$ ) and $s(n)=s(n-1)$ if $b(n)=0$; and $s(n)=-s(n-1)$ if $b(n)=1, n>0$, is such that $\{s,-s\} \in \mathcal{S}^{3}$.

This result provides a very simple and practical way of transforming $S^{3}$ sequences into $S D$ and vice versa. In the first works on PDOs, the method used to obtain the oscillation frequency consisted on calculating the FFT of the bitstream in order to see where the maximum was placed. This method, although it provided the desired results, was very expensive in computation time and resources. Once the bitstream has been converted to a $S D$ sequence, it is very simple to obtain the oscillation frequency (with decimation and filtering).

Simulation and experimental results confirm that applying the above conversion, as an intermediate step, it is possible to extract the oscillation frequency directly from general PDO bitstreams. This conversion will be used later in Section V to obtain the oscillation frequency of the MEMS resonator directly from the bitstream of the PDO embedded in a chemical sensor.

\section{SUFFICIENT CONDITIONS FOR OSCILLATION AT THE RESONANT FREQUENCY OF THE RESONATOR}

1) Case $f=\frac{M}{N}, \rho=0$ :

Definition 3: A $b \in \mathcal{B}^{L}$ is said to be consistent with $u \in \mathbb{C}$ if $\mathbf{b}=\left(\operatorname{sgn}\left(p^{-1} u\right), \cdots, \operatorname{sgn}\left(p^{-L} u\right)\right)$.

Definition 4: A lossless oscillator is said to be tuned to a rational frequency $f=\frac{M}{N}$, with g.c.d. $(M, N)=1$, if for $\operatorname{sign}(\delta)=1$ and:

- $N$ even: $\left(\left\lfloor\frac{N}{4}+\frac{1}{2}\right\rfloor-(L+1) M\right) \bmod N=0$. - $N$ odd: $\left(\left\lfloor\frac{N}{2}+\frac{1}{2}\right\rfloor-(L+1) 2 M\right) \bmod 2 N=0$. 


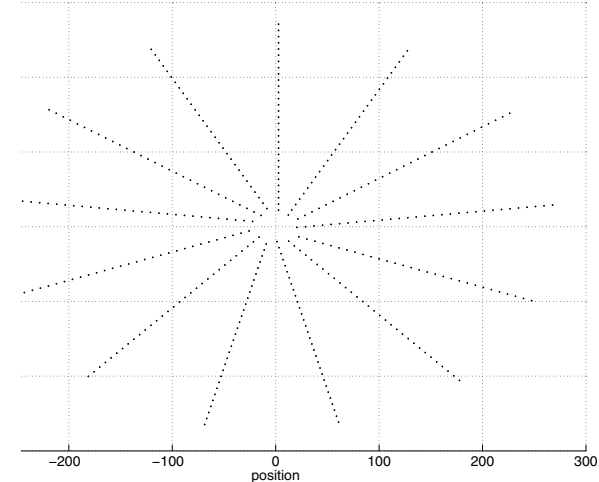

Fig. 2. Simulation of an oscillator with $L=1, \rho=0, f=5 / 13, \delta=$ -1 , with initial condition $u=20 e^{j \pi 13 / 45}$.

or for $\operatorname{sign}(\delta)=-1$ :

$-N$ even: $\left(\left\lfloor\frac{3 N}{4}+\frac{1}{2}\right\rfloor-(L+1) M\right) \bmod N=0$

- $N$ odd: $\left(\left\lfloor\frac{3 N}{2}+\frac{1}{2}\right\rfloor-(L+1) 2 M\right) \bmod 2 N=0$

It may be proved the following result, [2]:

Theorem 2: If a lossless oscillator is tuned to $f=\frac{M}{N} \in \mathbb{Q}, u \in \mathbb{C}$ is such that $\left|\operatorname{Real}\left(p^{n} u\right)\right|>$ $n|\delta|, 0 \leq n<N$ and $\mathbf{b}$ is consistent with $u$, then $\operatorname{sgn}\left(T_{n}((u, \mathbf{b}))\right)=\operatorname{sgn}\left(p^{n} u\right), n \geq 0$.

As an example, we will find an oscillator tuned to $f=5 / 13$. To this effect we need to solve the conditions for tuning, and a solution is $\delta<0, L=1$. Figure 2 shows a simulation of this oscillator. As it can be seen, $S^{3}$ sequences are obtained.

2) Case $f=\frac{M}{N}, \rho>0$ : We need to define an auxiliary sequence:

Definition 5: Given a $u \in \mathbb{C}$ we define the sequence $h_{n}(u), n>0$ as:

$$
h_{n}(u)=-j \delta p^{n-1} \sum_{i=0}^{n-1} p^{-i} \operatorname{sgn}\left(p^{i-L} u\right)
$$

with $h_{0}(u)=0$.

The following result can be proved, [2]:

Theorem 3: If for a leaky oscillator, an initial condition of the resonator $u \in \mathbb{C}$ and a frequency $f=\frac{M}{N} \in \mathbb{Q}$, it is for $N$ even:

$$
\frac{\left\lfloor\left(\frac{1}{4}-\frac{\operatorname{Arg}(u)}{2 \pi}\right) N\right\rfloor}{N}=\frac{\left\lfloor\left(\frac{1}{4}-\frac{\operatorname{Arg}\left(h_{N}(u)\right)}{2 \pi}\right) N\right\rfloor}{N}
$$

and $N$ odd:

$$
\frac{\left\lfloor\left(\frac{1}{4}-\frac{\operatorname{Arg}(u)}{2 \pi}\right) 2 N\right\rfloor}{2 N}=\frac{\left\lfloor\left(\frac{1}{4}-\frac{\operatorname{Arg}\left(h_{N}(u)\right)}{2 \pi}\right) 2 N\right\rfloor}{2 N}
$$

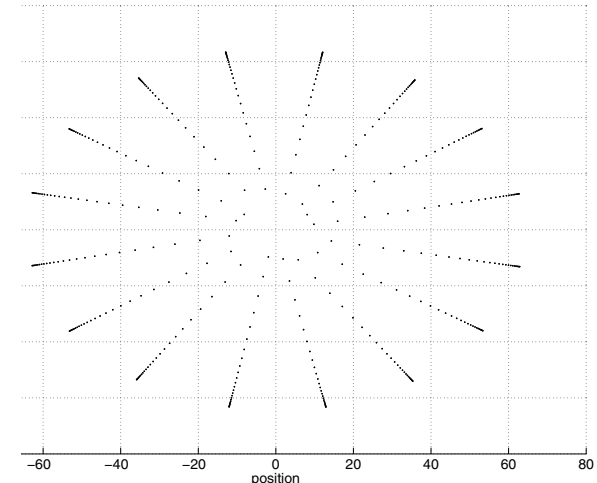

Fig. 3. Simulation of an oscillator with $L=3, \rho=0.005, f=$ $5 / 16, \delta=1$, with initial condition $u=10 e^{j \pi / 16}$.

and $u \in \mathbb{C}, n_{0} \geq 0$ are such that:

a) $\left|\operatorname{Real}\left(p^{n} u\right)\right|>|\delta| \frac{1-\alpha^{n}}{1-\alpha}, 0 \leq n<n_{0}$ and,

b) $\quad \frac{1-\alpha\left\lfloor\frac{n}{N}\right\rfloor N}{1-\alpha^{N}}\left|\operatorname{Real}\left(p^{n \bmod N} h_{N}(u)\right)\right|$

$|\delta| \frac{1-\alpha^{N}}{1-\alpha}, n \geq n_{0}$

then for $\mathbf{b} \in \mathcal{B}^{L}$ consistent with $u, \operatorname{sgn}\left(T_{n}((u, \mathbf{b}))\right)=$ $\operatorname{sgn}\left(p^{n} u\right), n \geq 0$.

An example of the behavior predicted by this result can be found in Figure 3. Condition b) of the result can be fulfilled for $\alpha \rightarrow 1$.

\section{DIRECT PDOS: VELOCITY FEEDBACK}

PDOs under certain conditions provide $S^{3}$ sequences [2]. The time evolution of a standard PDO is described by equation (4).

The usual interpretation with a mechanical resonator is that the PDO senses information on the position of the resonator and applies a delta of force after $L$ clock cycles. This force is translated into an instantaneous change in the velocity of the resonator. Usually the real part of the complex variable $u_{n}$ is the position of the resonator and its imaginary part is the velocity. It can be easily checked that:

$$
u_{k+n}-p^{n} u_{k}=-j \delta p^{n-1} \sum_{i=k}^{k+n-1} p^{-i} \operatorname{sgn}\left(u_{i-L}\right)
$$

On the other hand, in order to obtain $S^{3}$ sequences at the output of the PDO, for a lossless resonator with rational frequency, it is necessary that the oscillator is 'tuned' to this frequency, [2].

The reason to have frequencies to which the PDO is not 'tuned' is due to the fact each time the oscillator applies a delta of force to the resonator, it is not guaranteed in general that $\operatorname{sign}\left(\operatorname{Im}\left(u_{n}\right)\right)$ coincides with the sign of the applied excitation, which depends on the position, with 


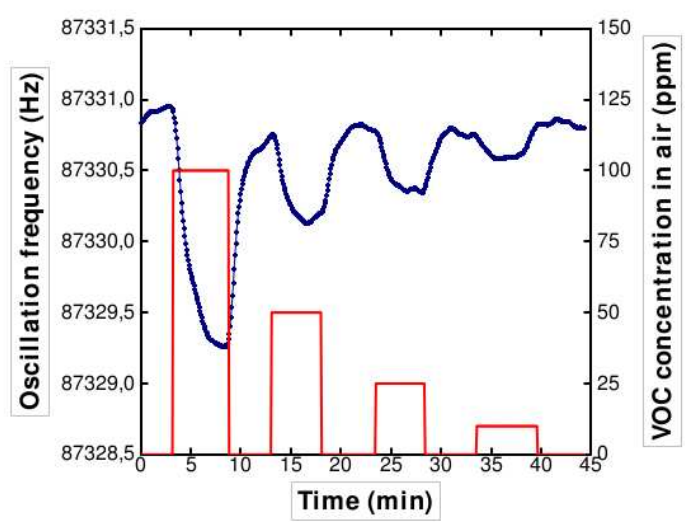

Fig. 4. Oscillation frequency of a MEMS cantilever with PDMS (Polydimethylsiloxane) as a function of time. Octane concentration is changed each 10 minutes.

a delay. In order to guarantee the coincidence we may define a variation of the typical PDO topology on which:

$$
u_{n+1}=p\left(u_{n}+\delta \operatorname{sgn}\left(u_{n}\right)\right)
$$

or

$$
u_{n+1}=p\left(u_{n}+j \delta \operatorname{sign}\left(\operatorname{Im}\left(u_{n}\right)\right)\right)
$$

For convenience, we use the first expression (13). This variant of the PDO will be called DPDO (Direct PDO). With this circuit, at each sampling time we must sense the sign of the variable that is instantly changed by the application of the delta of force. If we apply an excitation of the same sign $(\delta>0)$ we are increasing the energy of the resonator, and for the other sign $(\delta<0)$ we are stopping the resonator (unless the resonator is near the origin, $\left|u_{n}\right|<\delta$ ). This fact can be used in some applications, [4]. In practical realizations a few ns delay can be present.

The following results can be proved [3]:

Theorem 4: If a lossless DPDO of frequency $f=\frac{M}{N}$, g.c.d. $(M, N)=1$ has an initial condition $u_{0}$ such that $\left|\operatorname{Real}\left(p^{n} u_{0}\right)\right|>n|\delta|, 0 \leq n<N$, then $\operatorname{sgn}\left(u_{n}\right)=\operatorname{sgn}\left(p^{n} u_{0}\right)$.

In the case of a leaky resonator, in which $p=\alpha e^{j 2 \pi f}, 0<\alpha<1$, the following result can be obtained.

Theorem 5: There is a $0<\alpha_{1}<1$ such that for a leaky DPDO, with $1>|p|>\alpha_{1}$ and rational frequency, $f=\frac{M}{N}$,g.c.d. $(M, N)=1$, there is a constant $C(p) \in \mathbb{R}^{+}$ such that if $\left|\operatorname{Real}\left(p^{n} u_{0}\right)\right|>C(p), 0 \geq n \geq N-1$, then $\operatorname{sgn}\left(u_{n}\right)=\operatorname{sgn}\left(p^{n} u_{0}\right), n \geq 0$.

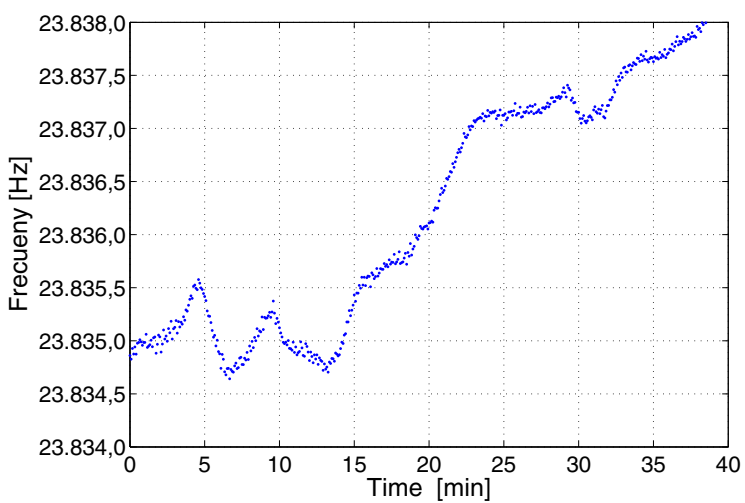

Fig. 5. Oscillation frequency as a function of time for random concentration of octane, obtained with a frequency meter.

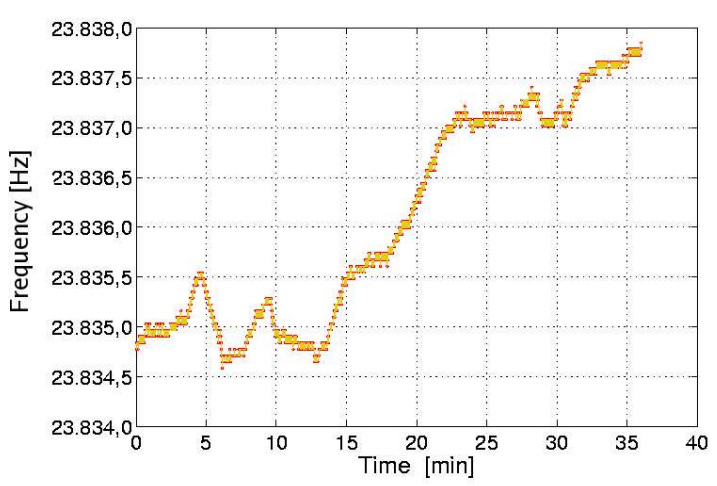

Fig. 6. Oscillation frequency obtained from the bitstream in the same experiment of Figure 5.

\section{Practical application: PDOS In CHEMiCAL SENSORS}

Due to its application to several fields such as safety, environmental detection, etc., the detection of volatile organic compounds, VOC, has been growing in interest during the last years.

To this effect, polymer layers deposited in micromachined resonators allow selective detection of volatile organic gas compounds (VOC). VOC molecules can be absorbed by the polymer layer, thus resulting in a change of its mass. Furthermore, if the polymer layer is deposited on the moveable arm of a MEMS device, the MEMS device will change its resonant frequency, following changes in gas concentration. In order to sense such small frequency changes, the MEMS resonator is imbued in the feedback loop of a PDO.

Figure 4 shows the oscillation frequency of the MEMS cantilever, on which a PDMS (Polydimethylsiloxane) polymer layer has been deposited, as a function of time. In this experiment the concentration of octane is changed each 10 minutes. The oscillation frequency has been obtained with a frequency meter. In contrast, Figures 5 and 6 show the oscillation frequency for a random succession of changes in gas concentration, used with 


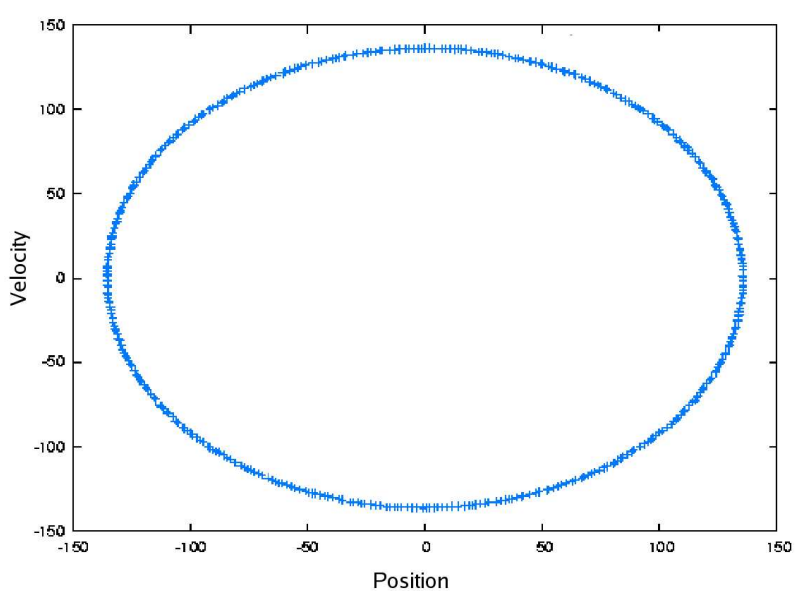

Fig. 7. One of the two limit orbits for a PDO with $f=0.22, L=0$ and a resonator with $Q=150$.

another MEMS resonator. The oscillation frequency in Figure 5 has been obtained with a frequency meter from the Wheadstone bridge of the MEMS cantilever. In this case, therefore, the oscillation frequency is obtained from the position analog waveform. Figure 6 shows the frequency obtained directly from the bitstream of the oscillator for the same experiment of Figure 5, applying the bitstream transformation shown in Section II and filtering. Although these are preliminary results, it can be observed that the digital oscillation frequency closely follows the variations present in Figure 5.

Finally, another open question of practical interest is whether the oscillation frequency of PDOs depends on the initial condition of the resonator. Preliminary simulation results indicate that for low losses resonators the oscillation frequency does not depend appreciably on the initial conditions of the resonator, although different limit orbits may exist. As an example Figure 7 shows a limit orbit obtained for $f=0.22$ and $Q=150, L=0$. Depending on the initial condition of the resonator (see Figure 8), this orbit is finally reached. Red points in Figure 8 reach the limit orbit of Figure 7, whereas white points reach a slightly rotated version of the orbit of Figure 7 . In both cases, though, the oscillation frequency is the same $\left(f_{\text {osc }}=0.21896955\right)$.

\section{CONCLUSiOnS}

Several rigorous results have been presented: PDOs can produce under some circumstance $S^{3}$ sequences at the resonant frequency of the resonator, these sequences are closely related to those of first-order sigma-delta modulators. The practical application of these circuits has been shown with a MEMS cantilever with PDMS as chemical sensor. A new variation of the standard PDO topology has also been presented on which the feedback variable is the speed of the MEMS resonator, instead of its position.

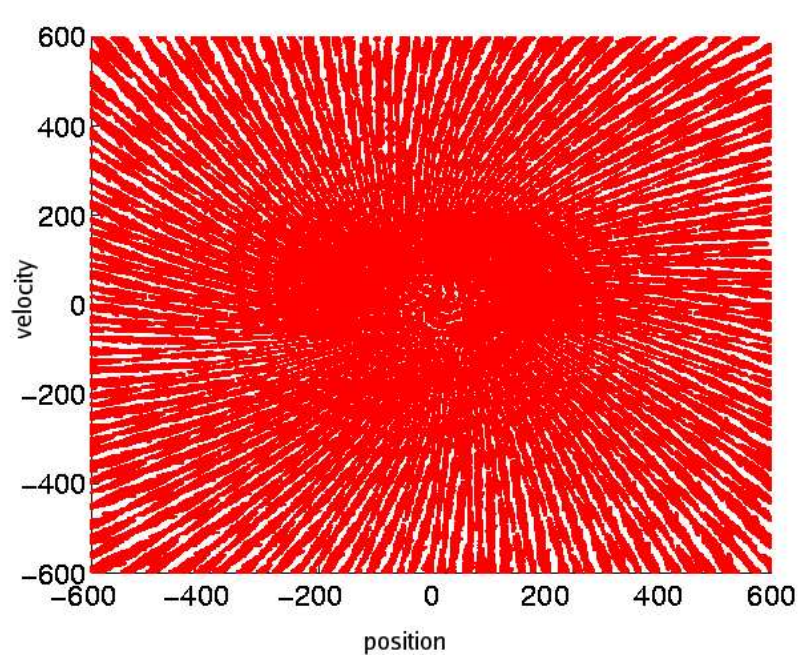

Fig. 8. With the same conditions as in Figure 7, partition of a rectangle of initial conditions of the resonator. Dark points reach a different limit orbit than white points. Both orbits have the same oscillation frequency.

\section{ACKNOWLEDGEMENTS}

This work was supported by CICYT DPI2004-06756C03-02 and CICYT TEC2007-67951/MIC.

\section{REFERENCES}

[1] Dominguez, M.; Pons-Nin, J.; Ricart, J.; Bermejo, A.; Costa, E.F.; Morata, M.; Analysis of the $\Sigma-\Delta$ pulsed digital oscillator for $M E M S$, IEEE Trans. on Circuits and Systems I, Vol. 52, No. 11, p. 2286-2297, 2005.

[2] M. Dominguez, J. Pons, J. Ricart General dynamics of Pulsed Digital Oscillators, IEEE Trans. on Circuits and Systems I, in print.

[3] M. Dominguez, Energy efficiency of pulsed actuations on MEMS resonators, submitted to IEEE Trans. on Circuits and Systems I.

[4] M. Dominguez, J. Pons, J. Ricart, Application of Pulsed Digital Oscillators in 'reverse mode' to eliminate undesired vibrations in high-Q MEMS resonators, Proc. ISCAS 2007, pp. 925 - 928, 2007.

[5] Czaplewski, D. A.; Dyck, C. W.; Sumali, H.; Massad, J. E.; Kuppers, J. D.; Reines, I.; Cowan, W. D.; Tigges, C. P.; A Soft-Landing Waveform for Actuation of a Single-Pole Single-Throw Ohmic RF MEMS Switch, Journal of Microelectromechanical Systems, Vol. 15, No. 6, pp. 1586-1594, 2006.

[6] Horsley, D.A.; Davis, W.O.; Hogan, K.J.; Hart, M.R.; Ying, E.C.; Chaparala, M.; Behrang Behin; Daneman, M.J.; Meng-Hsiung Kiang; Optical and mechanical performance of a novel magnetically actuated MEMS-based optical switch, Journal of Microelectromechanical Systems, Vol. 14, No. 2, pp. 274-284, 2005.

[7] Jin Qiu; Lang, J.H.; Slocum, A.H.; Weber, A.C.; A bulkmicromachined bistable relay with $U$-shaped thermal actuators, Journal of Microelectromechanical Systems, Vol. 14, No. 5, pp. 1099-1109, 2005.

[8] Leus, Vitaly; Hirshberg, Arnon; Elata, David; Optimizing the Dynamic Response of RF MEMS Switches using Tailored Voltage Pulses, International Conference on Thermal, Mechanical and Multi-Physics Simulation Experiments in Microelectronics and Micro-Systems, pp. 1 - 4, 2007.

[9] Gray, R.M. Spectral analysis of quantization noise in a singleloop sigma-delta modulator with DC input, IEEE Transactions on Communications, Vol. 37, p. 588-599, 1989. 